Annales Geophysicae (2001) 19: 425-434 (c) European Geophysical Society 2001

\title{
Super Dual Auroral Radar Network observations of fluctuations in the spectral distribution of near range meteor echoes in the upper mesosphere and lower thermosphere
}

\author{
N. F. Arnold, T. R. Robinson, M. Lester, P. B. Byrne, and P. J. Chapman \\ Department of Physics and Astronomy, University of Leicester, University Road, Leicester, LE1 7RH, UK
}

Received: 30 September 1999 - Revised: 26 July 2000 - Accepted: 12 December 2000

\begin{abstract}
The Doppler shifts of meteor echoes measured by the SuperDARN HF radar network have been used in several studies to observe neutral winds in the upper mesosphere and lower thermosphere region. In the absence of accurate height information for individual meteors, it has been necessary to assume a statistical mean meteor layer where the variations in altitude were not correlated to changes in the horizontal winds. Observations of spectral width distribution variations made by the radars allow an independent determination of the systematic error in the height. We have investigated the dependence of this distribution on a number of factors including the radar geometry, diurnal and seasonal cycles, variations in solar UV irradiance and geomagnetic activity.

Changes in the altitude of the mean meteor layer observed at different radar ranges provide us with some insight into the structure of the upper mesosphere and the lower thermosphere within which the meteors are being ablated. An examination of the spectral widths, as measured by the CUTLASS Finland radar, in the days preceding and following a Storm Sudden Commencement in April 1997, illustrates how the spectral properties of the observed region can be affected. The variations in the widths were consistent with model calculations of the changes to the temperature profile over this interval. Further refinements in the determination of the spectral width are outlined for future experiments.
\end{abstract}

Key words. Meterology and atmospheric dynamics (middle atmosphere dynamics; thermospheric dynamics; instruments and techniques)

\section{Introduction}

In a concerted effort to study global high-latitude convection flows in the ionosphere, an international consortium of research groups has collaborated on the construction of a chain of oblique sounding HF radars in both the Northern and

Correspondence to: N. F. Arnold (nfa1@ion.le.ac.uk)
Southern Hemispheres (Greenwald et al., 1995). One important feature of the SuperDARN network is that the radars are based on a single design and regularly run coordinated observational sequences with standard data processing techniques. This ensures a high level of consistency in the data between the instruments. The data from the HF radars also contains clutter from other sources. Backscatter from the ground via reflection from the ionosphere (e.g. Samson et al., 1996) and meteor scatter (Hall et al., 1997; Jenkins et al., 1998) often account for a significant fraction of the observations.

Meteor echoes provide an important tracer of neutral atmospheric conditions in the upper mesosphere and lower thermosphere as the plasma generated by the ablating meteors drifts with the neutral winds (see, for example, McKinley, 1961). Greenhow (1952) and Robertson et al. (1953) carried out the first observations of wind motions using this technique. Modern, dedicated meteor radars can determine the altitude of a meteor to within $1 \mathrm{~km}$ (Hocking et al., 1997).

The Northern Hemisphere SuperDARN radar chain is particularly suited to the task of monitoring large scale flows in the mesosphere directly above the winter stratospheric polar vortex. The circulation near the upper mesopause is affected both by upward propagating waves, such as planetary scale Rossby waves, and by changes in solar irradiance and high energy particle precipitation. There is the distinct possibility that these processes may have a persistent influence on the lower atmosphere through dynamical coupling, especially in the winter months (Arnold and Robinson, 1998). Whilst satellite observations can provide global coverage with good relative accuracy between measurements, the temporal resolution at any location is poor and the length of the time series is typically only a few years (Houghton et al., 1984). A number of dedicated upper atmosphere radars have been in operation for many years (e.g. Tetenbaum et al., 1986). However, different observational techniques tend to introduce intercomparison difficulties, and the number of instruments available at high latitudes is low.

The principal limitation on the value of meteor data from the SuperDARN radars to date has been the lack of detailed 


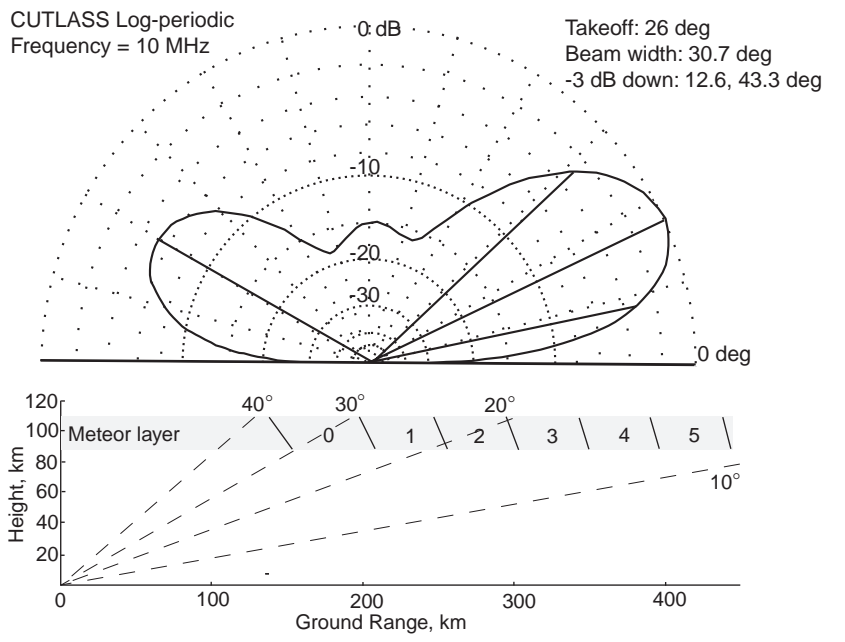

Fig. 1. (a) Log-periodic plot of the theoretical gain of the $10 \mathrm{MHz}$ CUTLASS radar transmitter as a function of elevation angle for optimum azimuthal pointing direction. Maximum gain has a take off angle of $26^{\circ}$ with a beam width of 30.7. (b) Intersections between the meteor ablation layer between $90-110 \mathrm{~km}$ and the six nearest SuperDARN range gates. Guide elevation angles in intervals of $10^{\circ}$ are denoted by dashed lines.

information about the altitude of the observations made. Hall et al. (1997) carried out a statistical intercomparison with the collocated MF radar at Saskatoon, but this could only restrict the possible effective altitude range to the region between $80-100 \mathrm{~km}$. Introducing a priori knowledge about the phase of the semi-diurnal tidal component, the altitude range could be narrowed to $94 \pm 3 \mathrm{~km}$. Assuming that the spectral widths were linearly dependent upon the ambi-polar diffusion, the authors were able to demonstrate that this height range was consistent with the peak and mean values of the spectral width distribution (91 and $97 \mathrm{~km}$, respectively). To arrive at this result, they used values of the ambi-polar diffusion coefficients taken from McKinley (1961). In their simple model, it was also assumed that the atmosphere did not vary with time.

It will be shown in the following sections of the paper that the height distribution of these spectral widths can vary significantly. Geometrical considerations related to the way the radars operate result in different distributions at each range gate. Temporal changes occur because the observed meteor ablation changes with the time of the day, the seasons and the occurrence of meteor showers. In addition, there will be changes in the upper mesosphere and lower thermosphere due to tides, seasonal forcing, solar irradiance variations and geomagnetic activity. Whilst the great majority of these changes fall within the experimental error quoted above, they are systematic and will bias the wind measurements, especially if there are significant vertical shears present between 91-97 km.

Section 2 of the paper examines the relationship between the spectral width and the radar range. Whether due to chan- ges in the properties of the meteors themselves or in atmospheric conditions, a number of processes that lead to a systematic variation in the spectral width distribution are identified in Sect. 3. In as much as the relationship between spectral width and ambi-polar diffusion is valid, one would expect to find the spectral width distribution to be temperature dependent. Section 4 outlines how the ambi-polar diffusion, as seen by the radars, could be affected by changes in the temperature.

We examine the possibility that the relationship between spectral width and altitude, combined with the sensitivity of the gain of the radar antennae to elevation angle, provides information about vertical structures in the upper mesosphere and lower thermosphere. An interval containing an episode of relatively high geomagnetic activity was examined in detail in Sect. 5. The spectral width changes before and after a Storm Sudden Commencement were consistent with those predicted by an empirical model that took into account the atmospheric temperature changes. The penultimate section discusses the limitations inherent in assuming that the spectral width can be associated with the ambi-polar diffusion coefficient and how future observations may be still further refined. A concluding section is provided at the end.

\section{Variation in spectral width with range}

The SuperDARN HF radars were designed to generate a narrow main lobe beam width of around $3^{\circ}$ through the constructive interference of 16 antennae (see, for example, Milan et al., 1997). Electronic changes in the phasing permits 'beam-steering', whereby a field of view of up to $45^{\circ}$ can be built up over a period of a few minutes. To investigate a large area of the auroral zones, the maximum gain of the radars was set at an oblique angle of approximately $25^{\circ}$ from the ground. The gain - elevation angle plot at a typical operating frequency of $10 \mathrm{MHz}$ (Fig. 1a) indicates that the gain falls off rapidly beyond the $-3 \mathrm{~dB}$ level at lower elevation angles and somewhat more slowly at higher angles. Within a cone of $18-40^{\circ}$, therefore, the response is quite uniform. Outside this area, weaker meteor trails will become progressively more difficult to detect. The radars can operate at frequencies ranging between 8-20 MHz. Each frequency produces a different gain - elevation response with implications that will be the subject of a follow-up study.

At $10 \mathrm{MHz}$, there are lobes in the backwards and verticallooking directions, but with significantly reduced gain sensitivities (the values shown are for transmit efficiency only; total return gain would double the effect on a logarithmic scale). The reverse lobe has a similar geometry to the front lobe and thus, the spectral width information would be similar in both directions (assuming that the distribution of meteors is isotropic). It is important to note that this backward view will affect the mean velocity, particularly in the meridionally pointing beams. The meteors from the upward looking lobe would register at a range of around $100 \mathrm{~km}$, which is usually too close to the radars to be detected. The default 
Table 1. Variations in total gain over the meteor ablation region for the first five range gates

\begin{tabular}{cccccccc}
\hline $\begin{array}{c}\text { Gate } \\
\text { Number }\end{array}$ & $\begin{array}{c}\text { Range } \\
\mathrm{km}\end{array}$ & \multicolumn{3}{c}{ Elevation Angle $\left.{ }^{\circ}\right]$} & \multicolumn{3}{c}{ Gain [dB] } \\
& $90 \mathrm{~km}$ & $100 \mathrm{~km}$ & $110 \mathrm{~km}$ & $90 \mathrm{~km}$ & $100 \mathrm{~km}$ & $110 \mathrm{~km}$ \\
\hline 0 & 180 & 30.0 & 33.7 & 37.7 & -0.28 & -1.08 & -1.75 \\
1 & 225 & 23.6 & 26.4 & 29.3 & -0.18 & +0.00 & -0.25 \\
2 & 270 & 19.5 & 21.7 & 24.0 & -1.26 & -0.56 & -0.22 \\
3 & 315 & 16.6 & 18.5 & 20.4 & -2.70 & -1.68 & -0.94 \\
4 & 360 & 14.5 & 16.1 & 17.8 & -4.18 & -3.02 & -1.98 \\
\hline
\end{tabular}

or 'common programme' observational mode makes its first range measurement at a distance of $180 \mathrm{~km}$.

In the common programme, the range data is divided into 75 regularly spaced intervals of $45 \mathrm{~km}$ each. By convention, the first range gate is numbered 0 . All of the SuperDARN radars operate together in this mode for at least half of the days of every month. Figure 1b shows the geometry of the first six range gates as they intersect the atmosphere at a nominal height range of $90-110 \mathrm{~km}$. Range gate 1 corresponds most closely to the optimum radar elevation angle at a frequency of $10 \mathrm{MHz}$. At gates 3-5, the 'meteor layer' begins to move outside the optimal viewing geometry and this will be most pronounced for the lowest altitude returns. Signal power falls off to the fourth power of distance (a doubling of the distance is equivalent to a drop off in sensitivity of 12 $\mathrm{dB}$.) As the meteors become relatively more difficult to detect at the furthest ranges, the differential sensitivity ensures that more meteors at $110 \mathrm{~km}$ are detected than at an altitude of $90 \mathrm{~km}$.

For the purposes of measuring meteor returns, it will be assumed that the rays will travel in straight lines because the ionospheric refraction is weak below the E-region. This will be less true for echoes from an altitude of $110 \mathrm{~km}$ and at further ranges, where the range-integrated electron concentration becomes more significant. However, the error introduced will still be considerably lower than other uncertainties in the technique, and may affect the determination of the range of the echoes rather than the spectral widths directly.

Table 1 outlines the variation in elevation angle and gain for the nearest six ranges at heights of 90, 100 and $110 \mathrm{~km}$ for the CUTLASS Finland radar at $10 \mathrm{MHz}$, excluding the correction to the gain as a function of increasing range. As mentioned above, the sensitivity at range gate 1 is fairly symmetrical about $100 \mathrm{~km}$, whereas at the nearest gate, there is a small loss in sensitivity at $110 \mathrm{~km}$, compared to $90 \mathrm{~km}$. At the further ranges, the situation is reversed with the enhanced sensitivity at higher altitudes. For those meteors closest to the radar, the power of the echoes is large enough that this small difference tends not to have a very marked effect on whether they are detected. At a distance of $360 \mathrm{~km}$, the signals are getting close to the noise threshold, as will be demonstrated later in this section and thus, there will be a significant reduction in the number of low altitude echoes observed, compared with those at greater heights.

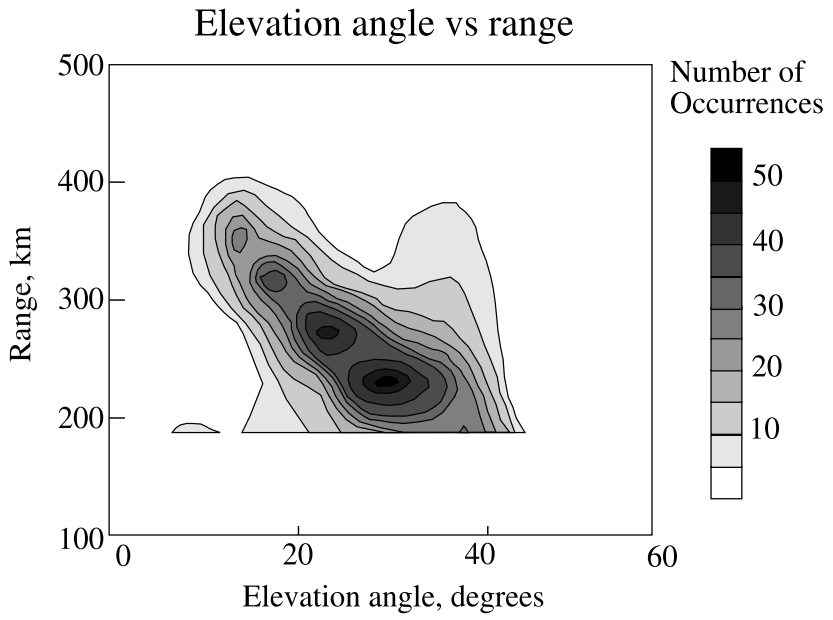

Fig. 2. Meteor occurrence distribution for the first six range gates of the CUTLASS Iceland radar as a function of elevation angle and range 02:00-04:00 UT, 11 November 1998.

Data from the interferometer on the CUTLASS Iceland East radar illustrates qualitatively the variation in the elevation angle of the meteor echoes with range at a frequency of $10 \mathrm{MHz}$ (Fig. 2). At near ranges, the spread in elevation angle exceeded $25^{\circ}$, making a quantitative estimate of the height of the reflection layer impossible. There was an ambiguity in the phase measurement at the nearest gate of around $30^{\circ}$ due to limitations in the processing (Milan et al., 1997). A weak secondary distribution, starting around 280 $\mathrm{km}$ range and $34^{\circ}$, is the mirror image of the main distribution. This feature is almost certainly caused by a signal from the backward looking lobe.

To determine the sensitivity of the spectral width distribution to range, 10 days of common programme data have been processed during October 1998. To provide independent samples, the data were divided into $2 \mathrm{~ms}^{-1}$ line-of-sight velocity intervals for all 16 beams. The distribution for each velocity interval was then normalised to remove any sampling biases. The results of this analysis for gates 1, 3 and 5 have been provided in Fig. 3. The peak and mean of the spectral width distributions increased from the nearest range gate to the furthest. Also, for the first few range gates, the trend was small, as the variations in the gain with eleva- 
(a)
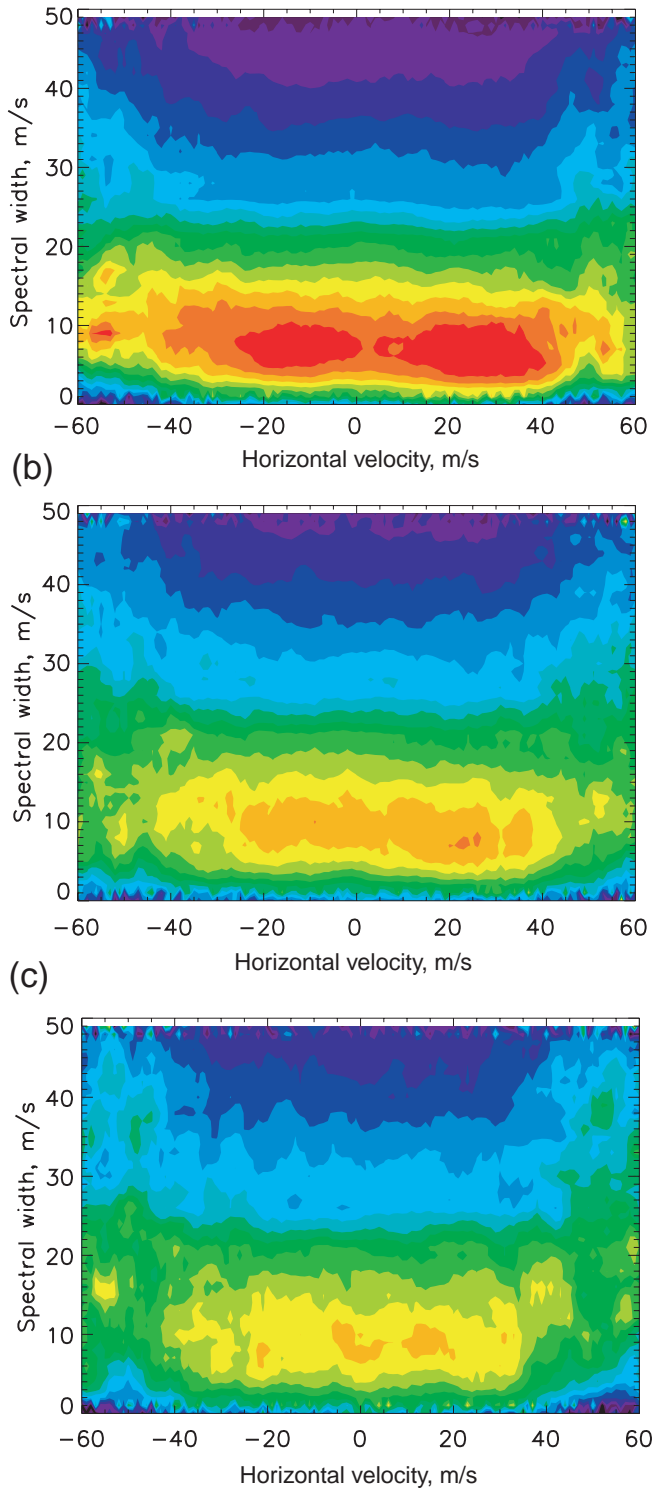

Relative Occurrence

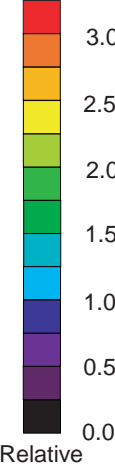

Occurrence
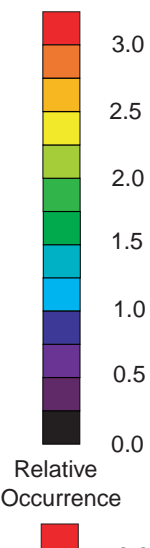

3.0 2.5

Fig. 3. Relative variations in meteor occurrences as a function of spectral width for velocity intervals between $\pm 60 \mathrm{~ms}^{-1}$ averaged over 15 common programme days in October 1998 from the CUTLASS Finland radar (a) gate 1 (b) gate 3 (c) gate 5.

tion angle were not yet very significant. However, at further ranges, the mean spectral widths shifted to higher values, as many weaker signals at low altitudes were lost. The distribution at further ranges was less smooth due to the reduction in the number of observations. On average, the spectral width increased by roughly $1 \mathrm{~ms}^{-1}$ per range gate, which corresponded to about a kilometre increase in altitude for the meteor ablation region.

In addition to the variation with range, we examined the sensitivity of the spectral width to power. Once the data was placed in the appropriate bins, the absolute occurrence rates were then normalised to give the relative distribution of
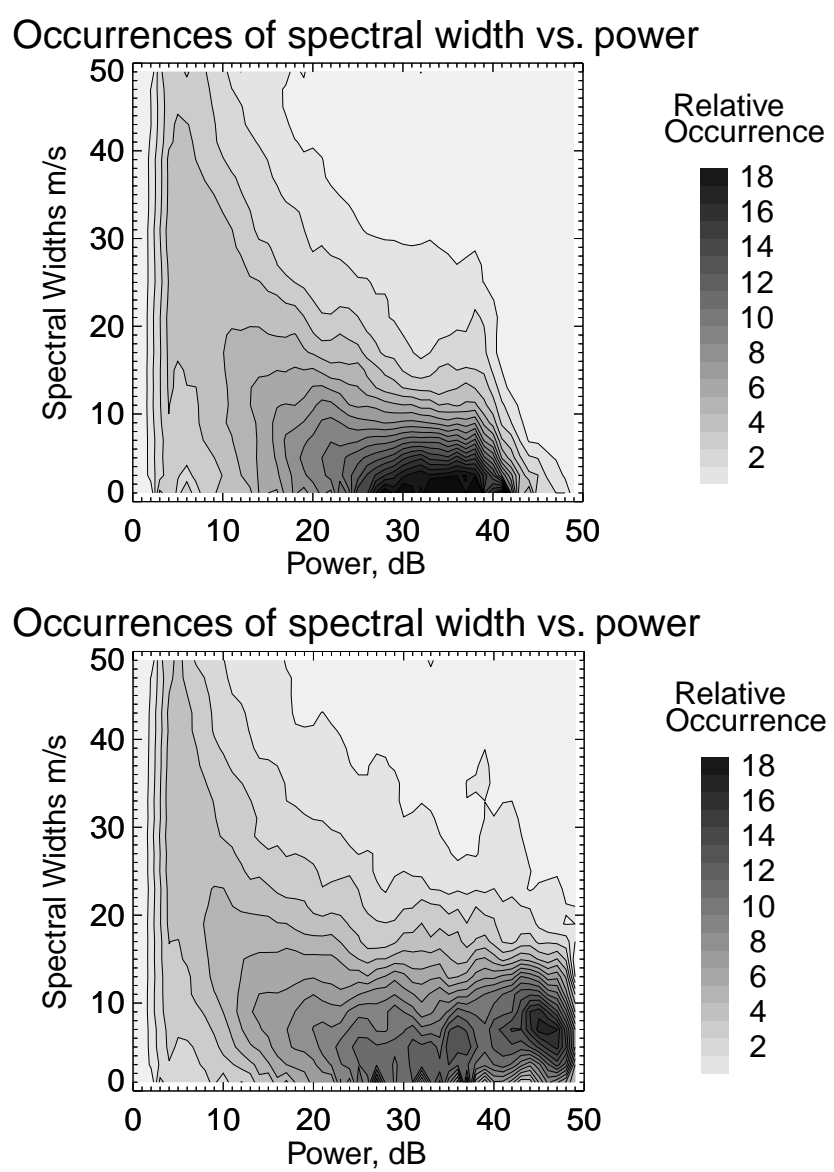

Fig. 4. Meteor occurrences from CUTLASS Finland as a function of power (dB) and spectral width $\left(\mathrm{ms}^{-1}\right)$, (a) 20-22 June 1999, (b) 2 July 1999.

widths for every unit of power and the results for 20-23 June 1999 are presented in Fig. 4a. The inverse relationship between spectral width and power for meteor echoes was due to the association of relatively high widths with greater altitudes and hence, further ranges. By way of comparison, 2 July 1999 in Fig. 4b, shows the impact of an episode of sporadic $E$. In the latter case, significant quantities of very high power and low spectral width occurrences could be identified. Below $30 \mathrm{~dB}$, there appeared to be much less contamination. It should be possible to utilise this relationship to minimise contamination from this source in future research.

\section{Statistical studies of variations in the spectral widths within the meteor ablation layer}

The CUTLASS Finland radar has been operational for over three years, during which the hardware and software have been gradually refined. It was necessary to assemble a statistically significant sample that was not so long that instrumental changes could become important. For example, from 29 October 1997 to the present, the day time operating frequency has been switched from $10 \mathrm{MHz}$ to $12 \mathrm{MHz}$ so that 
the number of ionospheric returns could be optimised as solar activity levels increased. Changes to the gain - elevation characteristics would introduce a bias into the processing of the data. Therefore, the period April - September 1997 was selected. Diurnal cycle, geomagnetic activity in the form of the Ap index, solar irradiance variations in the form of F10.7 $\mathrm{cm}$ solar radio fluxes and seasonal effects were examined to determine systematic trends in the spectral widths.

\subsection{Local time effects}

It has long been recognised that the meteor ablation region is influenced by the diurnal cycle (e.g. Weiss, 1959). The daily rotation of the Earth about its axis results in a variation in both the zenith angle of the incoming meteors and their velocity with respect to the atmosphere. The occurrences of spectral width common programme data for the month of April 1997 were binned in $2 \mathrm{~ms}^{-1}$ intervals and normalised for each two hour period of Universal Time. A further subdivision for each range gate was made to minimise the sampling problems associated with radar range, as mentioned in the previous section, especially during daylight hours when the number of observations is significantly reduced. Figure 5 shows the distribution for range gate 1 . There was a distinct maximum in the relative occurrence of spectral widths at around 10:00 UT where the mean width was $13 \mathrm{~ms}^{-1}$. This period corresponded to 13:00 Local Time. The occurrence peak at midnight was less pronounced, with a mean width of $18 \mathrm{~ms}^{-1}$. Assuming the relationship between ambipolar diffusion and height derived by Greenhow and Neufeld (1955) applied equally to spectral width, then the effective heights were 93 and $95 \mathrm{~km}$, respectively. This was in reasonable agreement with the $3 \mathrm{~km}$ diurnal variation obtained by Weiss. Similar distributions were obtained for the other range gates (not shown).

\subsection{Correlation with geomagnetic activity}

Geomagnetic influences on the high latitude neutral atmosphere at E-region heights around $110 \mathrm{~km}$ have been studied extensively (e.g. Dickenson et al., 1984). In addition to the direct impact that energetic particles have on the heat and momentum balance, neutral air parcels experience accelerating forces from the ionosphere and perturbations from waves propagating from the lower atmosphere (see, for example, Rees, 1989). In due course, the SuperDARN network will be employed to provide observations of the global scale motions that shape the response of the neutral atmosphere to geomagnetic variations. For the present study, only the direct impact of these variations on the spectral widths, as seen by the Finland radar, will be considered.

As outlined above, the spectral width is influenced by diurnal changes. Therefore, the following studies were carried out at 00:00-02:00 UT to minimise this effect. Since at least a quarter of the meteor occurrences were obtained during this interval, the size of the sample remained large. Days with unusually consistent and strong power returns at the nearest
Spectral width vs. Universal Time APRIL 97

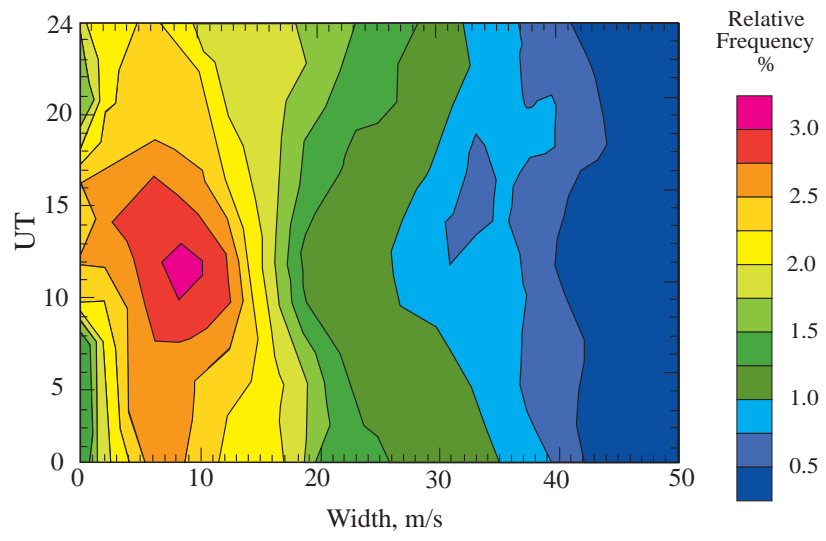

Fig. 5. Universal Time variations in spectral width distributions for the month of April 1997 from range gate 1 of the CUTLASS Finland radar. The distributions have been normalised for each time interval and the frequency of occurrence in each bin expressed as a percentage of the total.

ranges were rejected visually, as they were likely to be contaminated with Sporadic E. In the period between April and September, this left 116 days of usable common programme data. Range gate 1 was selected since there was no particular weighting given to any height and since it also produced a large number of echoes. Both the mean and median values of the spectral width were computed. It was found that the median gave the largest variation and would be less influenced by a relatively small number of high width contaminants. The geomagnetic activity levels were represented by the $A p$ index provided by the University of Göttingen.

Figure 6a shows the scatter plot of median spectral width against $A p$. The significance of the fit was computed to be better than $99.5 \%$. The width increased by roughly $1.1 \mathrm{~ms}^{-1}$ per 10 unit increase in the $A p$ index. There was considerable variance about the fit line, indicating that there were other factors that were important in determining the width. The atmospheric response to geomagnetic activity is itself nonlinear, as outlined above. A considerable component of the variation in the spectral widths was probably due to contributions from short period atmospheric wave phenomena, such as planetary and gravity waves and (non-diurnal) tides.

\subsection{Correlation with variations in extreme ultraviolet solar emissions}

Between April and September 1997, solar cycle activity was close to a minimum, with associated low values of $2800 \mathrm{MHz}$ (F10.7) solar radio fluxes and very weak variations over the 27-day rotation period. The radio flux is a good proxy for perturbations in high energy ultraviolet emissions from the Sun (see, for example, Hargreaves, 1992). F10.7 is only weakly correlated to $A p$ on time scales of less than 11 years, so the $A p$ contribution was not removed before carrying out the correlation. In September there was a significant flux en- 
(a)
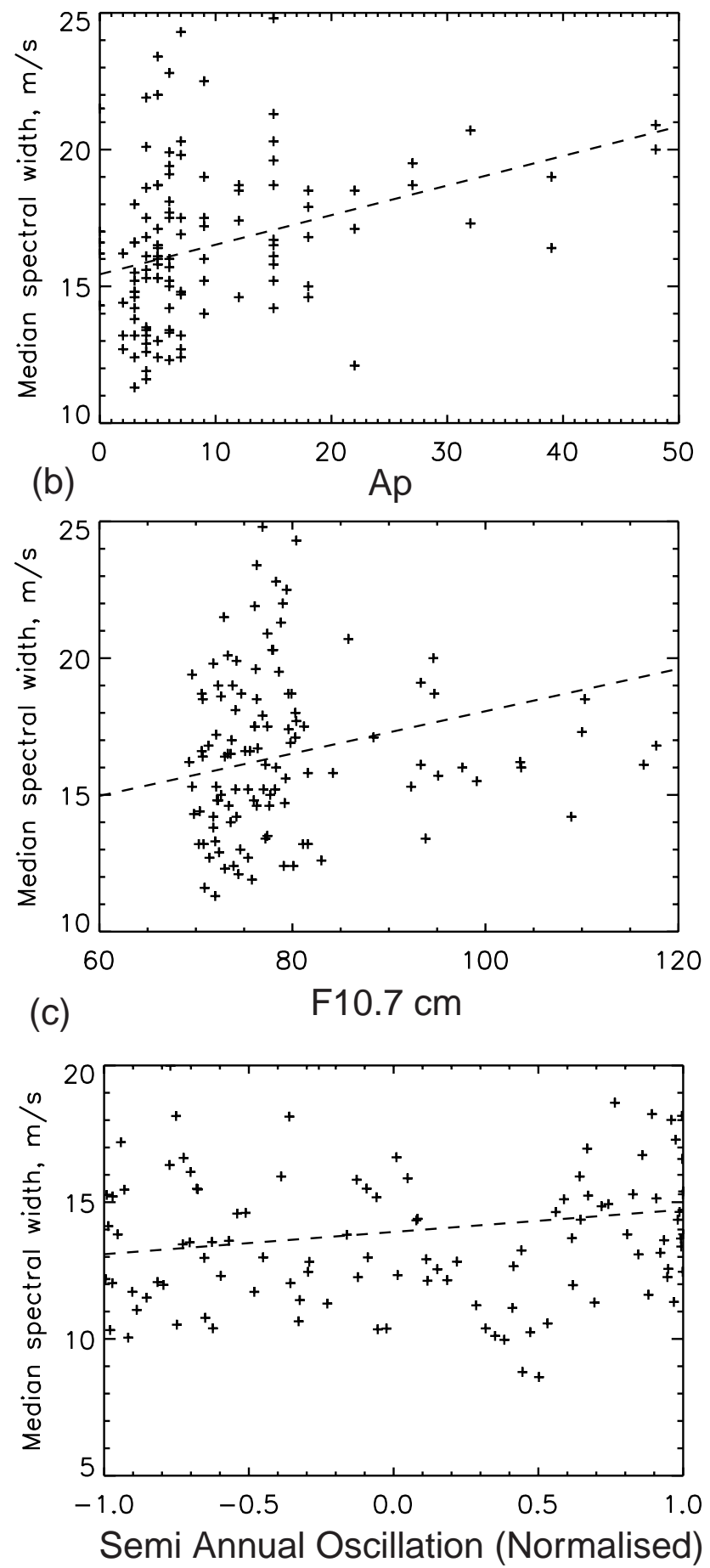

Fig. 6. Scatter plot of the median spectral width against several parameters for 0-2 UT between April and September 1997 using range gate 1 data from the common programme of the CUTLASS Finland radar. (a) Geomagnetic activity indicator $A p$, (b) solar flux F10.7 cm, (c) normalised amplitude of the semiannual oscillation.

hancement that almost doubled the emissions at the extremes of the solar electromagnetic emission spectrum.

The scatter plot (Fig. 6b) showed more variance than the geomagnetic activity due to the weaker dependence on ultraviolet irradiance changes. The correlation remained significant above the $99 \%$ level. The gradient of the fit predicted an increase in the spectral width of approximately $12 \mathrm{~ms}^{-1}$ between solar minimum and solar maximum. This is probably an overestimate due to the limited range of F10.7 data available in the study and the gradient calculation will be revisited in a few years when the solar activity level has reached its peak.

\subsection{Seasonal variations}

The F10.7 cm flux data excludes the seasonal component due to the eccentricity of the Earth's orbit around the Sun. In addition to orbital variations, the inclination of the Earth's axis with respect to the ecliptic plane produces an annual change in the insolation rates at the top of the atmosphere above the radar. To investigate possible annual and semiannual influences on the spectral width distribution, the data was cross-correlated with two idealised sinusoids with periods of 365.24 and 182.62 days, respectively. As the correlation is insensitive to multiplicative constants, the SAO was assigned unit maximum amplitude. The contributions to the spectral width variability ascribed to $A p$ and F10.7 were first removed from the data set.

The statistical significance of the correlation using an annual cycle did not exceed $70 \%$ for any lag during the year. The significance rose to greater than $95 \%$ when the data was correlated with a semi-annual oscillation whose peak amplitude occurred on 15 June. In Fig. 6c, the radar spectral widths have been plotted against the corresponding amplitude of the idealised SAO on those days, such that the lag produced the optimal fit described above. The amplitude of the peak-to-peak variation in spectral width from the resulting best straight-line fit was calculated to be $3.2 \mathrm{~ms}^{-1}$.

The empirical MSIS-90E (Mass Spectrometer Incoherent Scatter) empirical model (Hedin, 1983, 1991) was used to interpret these findings. Figure 7 illustrates the MSIS temperature structure between $90-110 \mathrm{~km}$. The maximum amplitude of the predicted semi-annual temperature oscillation over Finland was 3 June and this compared quite favourably with the radar observations. Below $100 \mathrm{~km}$, there was a strong annual variation with a minimum in June, whilst above, there was a maximum. Therefore, a radar that makes observations across this boundary will find the two competing effects neutralising each other, leaving the smaller semiannual cycle to dominate at this height. The data set will need to be extended over several years in order to enhance the prospects of extracting an annual cycle variation. Alternatively, as discussed in Sect. 2, by considering spectral widths from further range gates, the effective meteor detection height could be raised. 


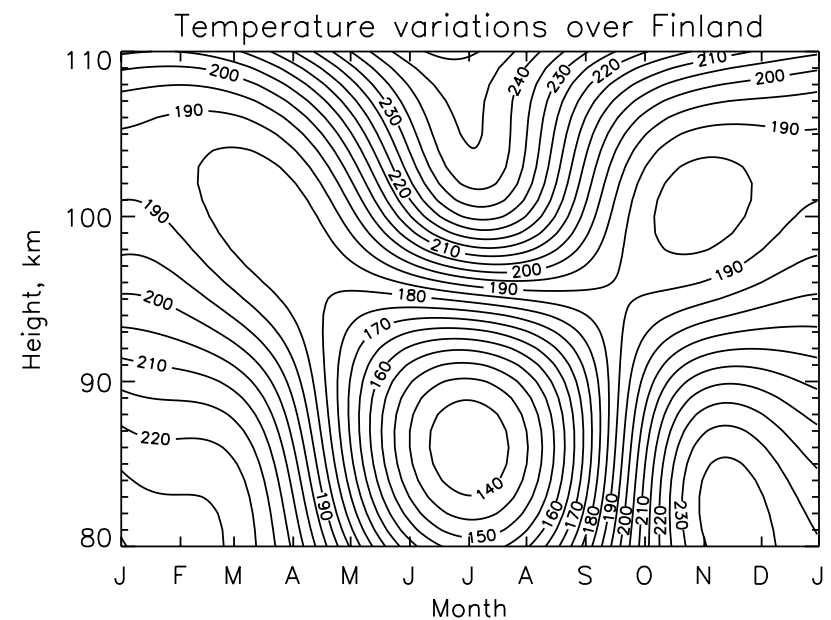

Fig. 7. MSIS-90 climatological temperatures over Hankasalmi between 90 and $110 \mathrm{~km}$.

\section{An approximate relationship between mean spectral width and temperature as measured by the Super- DARN radars}

Recently, Hocking (1999) has been able to obtain a statistical estimate of the temperature of the meteor region by examining the variation in the rate of decay of the meteors at a given height. The SuperDARN radars can neither pinpoint the position of a single meteor nor provide a measure of their decay rate in their present configurations. However, information about the atmospheric temperature is contained within the spectral width measurements. The spectral width is related to the ambi- polar diffusion coefficient, $D_{a}$ such that

$\Delta v=\frac{8 \pi D_{a}}{\lambda}$

where $\Delta v$ is the spectral width in Doppler units and $\lambda$ is the radar wavelength (Hall et al., 1997).

The diffusion coefficient determines the rate at which the meteor echoes decay, such that the power $P$ at time $t$ is given by

$P(t)=P(0) \exp \left(\frac{-\lambda^{2} t}{32 \pi^{2} D_{a}}\right)$

$D_{a}$ is given by

$D_{a} \propto \frac{T_{e}+T_{i}}{v_{i n}}$

where $T_{e}$ is the electron temperature, $T_{i}$ is the ion temperature and $v_{i n}$ is the ion-neutral collision frequency (Rees, 1989). In the meteor ablation region, collisions are sufficiently frequent so that the plasma temperature is similar to that of the surrounding air. To a first approximation, the temperature profile around the mesopause, where most of the meteors are detected, is isothermal. Under these conditions, the ambi- polar diffusion coefficient increases exponentially with height. Hocking (1999) expressed Eq. (2) in terms of pressure $p$ and temperature $T$

$D_{a} \propto \frac{T^{2}}{p}$.

It will prove useful to express the diffusion as a function of air density $\rho$, instead of pressure, because the ionisation rate, $q$, produced by the ablating meteor, is directly proportional to this quantity. From McKinley (1961),

$q=\beta \frac{\Lambda A}{2 \zeta \mu}\left(\frac{m}{\rho_{m}}\right)^{2 / 3} \rho V^{2}$

where $\beta$ is the probability that a single ablated atom with a mass $\mu$ would produce a free electron upon collision with an air particle; $\Lambda$ is the dimensionless heat-transfer coefficient; $A$ is the dimensionless shape factor that gives the effective cross sectional area; $m$ is the mass of the meteor; $\rho_{m}$ is its density; $V$ is the velocity with respect to the air parcel and $\zeta$ is the heat of ablation. Using the equation of state, diffusion has a functional relationship with density such that

$D_{a} \propto \frac{T}{\rho}$.

Thus, on a constant atmospheric density surface, the diffusion coefficient is linearly proportional to temperature. When the atmosphere expands and contracts, the height and pressure distribution of the meteor layer will vary accordingly, but the layer will be constrained to follow isopycnals, which are uniform density surfaces and are often used in the study of atmospheric dynamics (see for example Mcllveen, 1986).

As has been discussed in Sect. 2, the likelihood of detection of meteors by the SuperDARN radars is relatively insensitive to small changes in the altitude of the echoes, especially at the nearest ranges. Therefore, variations in the spectral width distribution at constant density can be attributed to changes in temperature or to changes in the properties of the meteors themselves. A statistically significant sample of meteors will form a well-defined distribution as a function of density and area within the field of view.

The distribution of the incoming meteors themselves evolves over time. For example, during meteor showers, the number of small meteors detected at relatively high altitudes increases significantly. Such episodes are relatively infrequent and typically last for only a few hours. Shower intervals can be removed from the study, if necessary, by noting the sudden dramatic increase in meteor occurrence rates. Similarly, diurnal and seasonal variations in the distribution profile can be taken into account. For much of this paper, very large numbers of meteors under a wide range of conditions were considered. The remaining uncertainties in the distribution caused by variations in meteor activity should be of a predominantly random nature with respect to the atmospheric changes under consideration. 


\section{PLANETARY MAGNETC THREE-HOUR-RANGE INDICES}

\section{Kp from 13 - 19 April 1997}
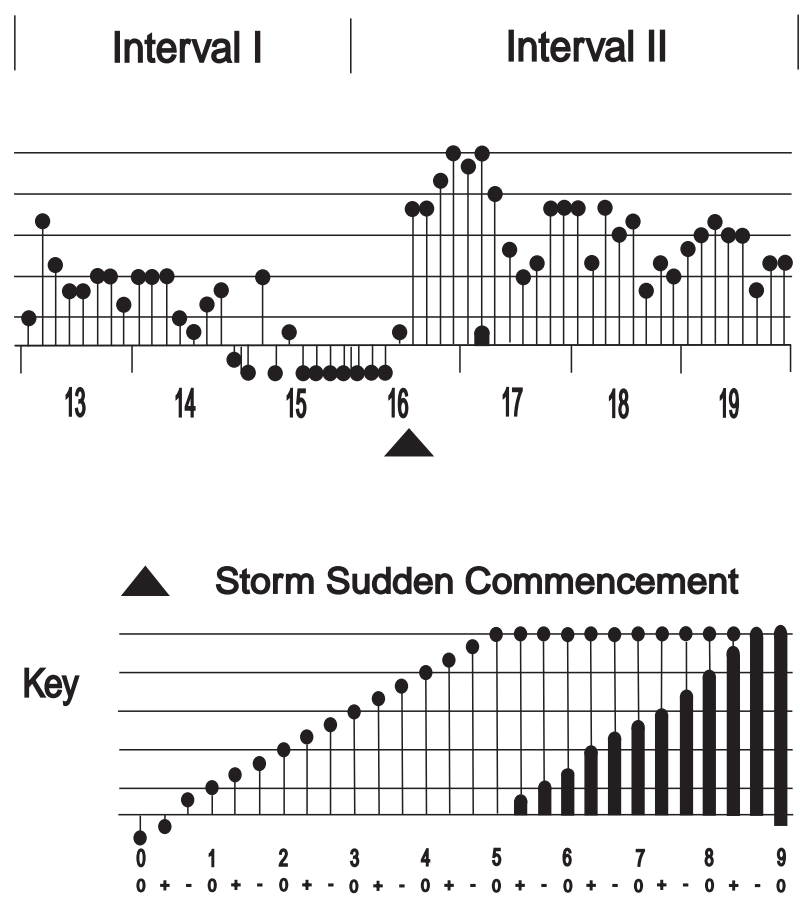

Fig. 8. Planetary 3-hour range indices $(K p)$ for 13-19 April 1997.

\section{Variations in spectral width before and during a geo- magnetically disturbed interval: a case study}

An interval of CUTLASS Finland data was selected with common programme coverage that contained a period of relatively low geomagnetic activity followed by one of enhanced activity. A Storm Sudden Commencement (SSC) marked the boundary between the two periods. Figure 8 shows the 3hourly planetary magnetic indices for the period 13-19 April 1997. The $K p$ values were converted to $A p$ so that they could be averaged and introduced into the empirical MSIS model at a later stage. The 13th to the 16th of the month formed the first interval and achieved an equivalent $A p$ index of 3.5, whilst the last four days made up the second interval with an equivalent $A p$ index of 15.8 .

The spectral width distributions of the meteor echoes for the first and fourth range gates for each of the intervals were normalised to take account of the different occurrence rates. The distributions obtained for the geomagnetically quiet interval can be found in Fig. 9a. There is a distinct shift in the peak and mean spectral width values between the two ranges, as described in Sect. 2. The difference in occurrence rates between the two gates, as a function of spectral width, was then expressed as a percentage with respect to the occurrence rate at gate four (Fig. 9b).

For the low $A p$ interval, the variation in spectral widths be- (a)

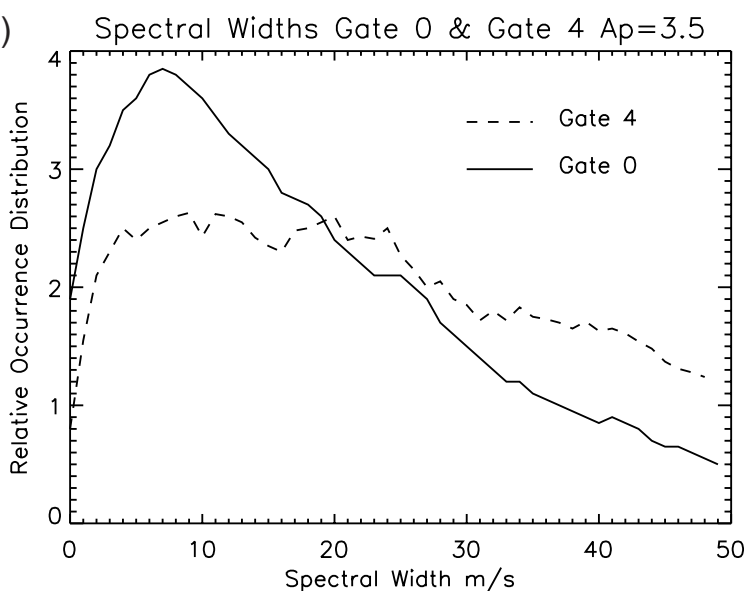

(b) Relative spectral width differences gate 4 - gate 0

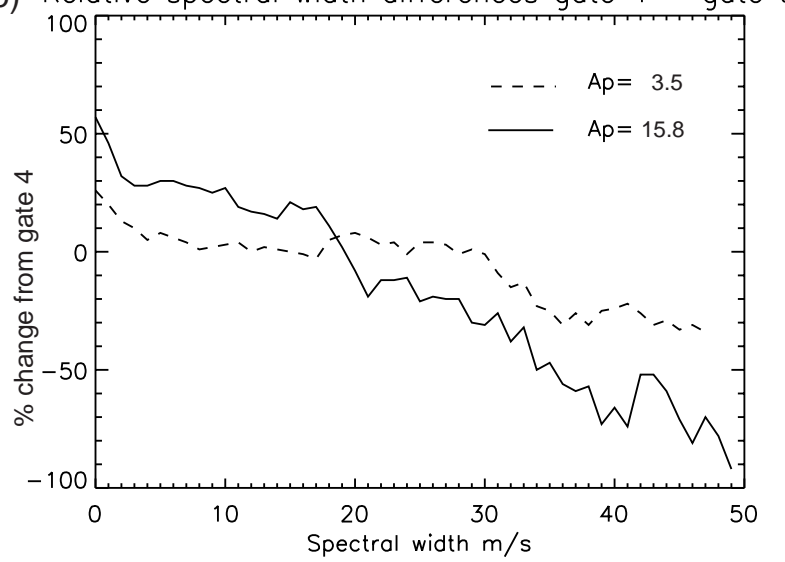

Fig. 9. (a) Distribution of meteor occurrence as a function of spectral width for range gate 0 (-) thick line and range gate 4 (- -) thin line during quiet geomagnetic conditions. (b) Relative differences between spectral width distributions gate 0 and gate 4 for $A p=3.5$ (- -) dashed line and $A p=15.8$ (-) whole line.

tween the near and far ranges was noticeable only at low and high values of spectral width, whilst between 5 and $30 \mathrm{~ms}^{-1}$, the relative change in spectral width was less than $10 \%$. For the interval following the SSC, the variation was much more pronounced for all widths. Either the radar was observing meteors from two distinct atmospheric layers, or the properties of the plasma had been affected by the onset of the geomagnetic storm. To test the first of these possibilities, the MSIS empirical model was again employed.

The climatological vertical temperature profile over Hankasalmi for the month of April 1997 was computed and plotted in Fig. 10a with the assumption that there was no geomagnetic activity in that period. The model predicted that the mesopause should be at an altitude of around $95 \mathrm{~km}$ with a minimum temperature of $172 \mathrm{~K}$. Above the mesopause, the temperature increased quickly to nearly $260 \mathrm{~K}$ in the space of just over $15 \mathrm{~km}$. Below, the temperature increases more gradually with a lapse rate of less than $-2 \mathrm{~K} / \mathrm{km}$. The on- 

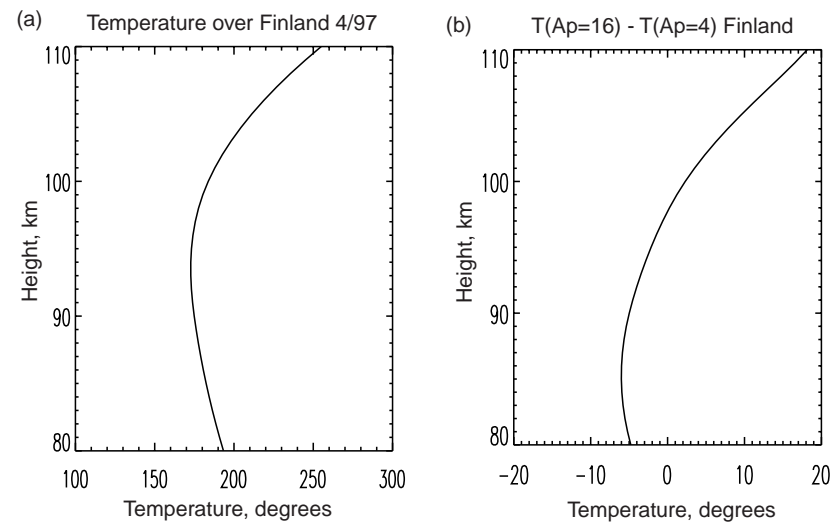

1997).

The spectral width distribution of the meteors will be affected by the presence of large numbers of over-dense meteors. Hall et al. (1997) estimated their numbers to be between 1 and $10 \%$ of the total. This number could be expected to rise at further ranges where weaker echoes can be lost. Within range gate 6 , the majority of the meteors have gains in excess of $10 \mathrm{~dB}$ above noise, so the ratio of over- to under-dense echoes should be relatively constant. As the number of these echoes falls off in proportion to their intensity, the majority of these echoes will spend only a small fraction of their time in the saturated state and the remainder as under-dense trails. The radar will produce a weighted average of these two stages to produce a spectral width that is somewhat reduced compared to its purely under-dense counterpart. This could result in an attendant underestimate of the effective height of the echoes and an error in the spectral width distribution with height. However, this would only become a serious problem in the current study if there was a link between the atmospheric temperature changes and the ratio of over- to under-dense echoes, as we are more concerned with relative changes to the width distribution. Clearly, this would be the case over diurnal and seasonal cycles as well as during meteor showers, but the errors should cancel out under other circumstances.

In this study, only common programme data has been used. However, a number of modifications can be introduced using observing time, which is set aside on the radars for nonstandard experiments; this will enhance the usefulness of the meteor observations. Firstly, the nearest range can be reduced to $60 \mathrm{~km}$ to capture meteors in side lobes that have high elevation angles. Secondly, the range resolution can be increased from $45 \mathrm{~km}$ to $15 \mathrm{~km}$, and the time integration reduced from 7 seconds to 2 seconds to reduce the incidence of multiple meteors in a single measurement. This would also help to identify 'non-grainy' echoes that are probably not meteors. As typical meteor trails are highly localised and usually only last for less than a second, there should not be a significant reduction in the signal-to-noise ratio.

A follow-up study using multiple frequencies will attempt to establish an optimum operating frequency (or indeed combination of frequencies). Since the antenna gain patterns change at different frequencies, the sensitivity to different heights will also change. According to McKinley (1961), lower frequency radars measure more echoes and the altitude of peak occurrence increases with wavelength. On the other hand, lower frequencies are more susceptible to propagation delays, D-region absorption and side-lobe contamination.

The CUTLASS radars are currently being modified to allow two independent signals to be transmitted and received at once (StereoCUTLASS). This change will effectively double the sensitivity of the radar to meteor echoes, which is especially useful in the daytime, when the count rate can be relatively low. Alternatively, it will allow part of the radar to continue to make near range scans at a time when the remainder is carrying out some other experiment at far ranges where no meteor detection is possible, thus, ensuring conti- 
nuity of coverage. A further option would be to illuminate the same volume of the sky at several frequencies simultaneously to provide additional information about its altitude through differences in the frequency dependent elevation gain characteristics.

\section{Conclusion}

The SuperDARN HF radar network can measure neutral winds near the mesopause using the Doppler shift of meteor trails from the atmosphere. We have shown that the height at which the winds are determined depends on both the radar geometry and the prevailing atmospheric conditions. An understanding of the geometry of the SuperDARN radars provides a sensitive means of monitoring changes in the mesopause region as it responds to geomagnetic activity and other phenomena over time scales of a day or more. A number of modifications to the observational programme of the network would enhance the quality of the meteor data, albeit at the expense of interfering with other scientific requirements. The introduction of StereoCUTLASS at the Iceland East and Finland sites will largely overcome this obstacle.

Information about the altitude, or at least variations in altitude, of the meteor echoes is of great importance if long term data sets of upper mesosphere and lower thermosphere winds are to be constructed. These winds provide insight into how the atmosphere responds to changes in solar and possibly human influences. In the absence of these corrections, there would remain an uncertainty about whether there had been a change in the magnitude of the winds over time or a change in the height of the observations. In addition, changes in the operation of the radars themselves over time could also introduce systematic errors if they are not taken into account.

Acknowledgements. The authors wish to thank the staff at the Radio and Space Plasma Physics Group at Leicester University for building and operating the CUTLASS radars and in particular to $S$. E. Milan and E. C. Thomas for a number of helpful discussions. We also note the helpful suggestions of the referees. The solar geophysics data was provided by the University of Göttingen via the World Data Center-A for Solar Terrestrial Physics, NOAA E/GC2, 325, Broadway, Boulder, Colorado, 80303, USA. N. F. Arnold was supported by an Advanced Research Fellowship from the Particle Physics and Astronomy Research Council of the United Kingdom.

The Editor-in-Chief thanks a referee for his help in evaluating this paper.

\section{References}

Arnold, N. F. and Robinson, T. R., Solar cycle changes to planetary wave propagation and their influence on the middle atmosphere circulation, Ann. Geophysicae, 16, 69-76, 1998.

Dickenson, R. E., Ridley, E. C., and Roble, R. G., Thermospheric general circulation with coupled dynamics and composition, J.
Atmos. Sci., 41, 205-19, 1984.

Greenhow, J. S., Characteristics of radio echoes from meteor trails, Proc. Phys. Soc. London, 65B, 169-174, 1952.

Greenhow, J. S. and Neufeld, E. L., The diffusion of ionized meteor trails in the upper atmosphere, J. Atmos. Terr. Phys., 6, 133-140, 1955.

Greenwald, R. A., Baker, K. B., Dudeney, J. R., Pinnock, M., Jones, T. B., Thomas, E. C., Villain, J.-P., Cerisier, J.-C., Senior, C., Hanuise, C., Hunsucker, R. D., Sofko, G., Koehler, J., Nielsen, E., Pellinen, R., Walker, A. D. M., Sato, N., and Yamagishi, H., DARN/SuperDARN: A global view of the dynamics of highlatitude convection, Space Sci. Rev., 71, 761-796, 1995.

Hall, G. E., MacDougall, J. W., Moorcroft, D. R., and St.Maurice, J.-P., Super Dual Auroral Network observations of meteor echoes, J. Geophys. Res., 102, 14603-14614, 1997.

Hargreaves, J. K., The solar-terrestrial environment, Cambridge University Press, pp 420, 1992.

Hedin, A. E., A revised thermospheric model based on mass spectrometer and incoherent scatter data: MSIS-83, J. Geophys. Res. 88, 10170-10188, 1983.

Hedin, A. E., Neutral atmosphere empirical model from the surface to the lower thermosphere: MSISE, J. Geophys. Res., 96, 1159$1172,1991$.

Hocking, W. K., Thayaparan, T., and Jones, J., Meteor decay times and their use in determining a diagnostic mesospheric 39pressure parameter: methodology and one year of data, Geophys. Res. Lett., 24, 2977-2980, 1997.

Hocking, W. L., Temperatures using radar-meteor decay times, Geophys. Res. Lett., 26, 3297-3300, 1999.

Houghton, J. T., Taylor, F. W., and Rodgers, C. D., Remote sounding of atmospheres, Cambridge University Press, pp 343, 1984.

Jenkins, B., Jarvis, M. J., and Forbes, D. M., Mesospheric wind observations derived from Super Dual Auroral Radar Network (SuperDARN) HF radar meteor echoes at Halley, Antarctica: preliminary results, Rad. Sci., 33, 957-966, 1998.

McIlveen, J. F. R., Basic meteorology: a physical outline, Van Nostrand Reinhold, Wokingham, pp 457, 1986.

McKinley, D. W. R., Meteor Science and Engineering, McGrawHill, New York, 1961.

Milan, S. E., Jones, T. B., Robinson, T. R., Thomas, E. C., and Yeoman, T. K., Interferometric evidence for the observation of ground backscatter originating behind the CUTLASS coherent HF radar, Ann. Geophysicae, 15, 29-39, 1997.

Rees, M. H., Physics and Chemistry of the Upper Atmosphere, Cambridge University Press, pp 289, 1989.

Robertson, D. S., Lilly, D. J., and Elford, W. G., Measurements of winds in the upper atmosphere by means of drifting meteor trails I, J. Atmos. Terr. Phys., 4, 255-274, 1953.

Roble, R. G. and Ridley, E. C., A thermosphere-ionospheremesosphere-electrodynamics general circulation model (TIMEGCM): Equinox solar minimum simulation, 30-500 km, Geophys. Res. Lett., 21, 417-420, 1994.

Tetenbaum, D., Avery, S. K., and Riddle, A., Observations of mean winds and tides in the upper mesosphere during 1980-1984, using the Poker Flat, Alaska, MST radar as a meteor radar, J. Geophys. Res., 91, 14539-14555, 1986.

Weiss, A. A., The temporal variation of the heights of reflection points of meteor trails, Aust. J. Phys., 12, 116-126, 1959. 